\title{
A Simple LED based on WiFi Environment Design and Implementation of Lighting System
}

\author{
Xiong Qiangqiang1, a , Liu Jianpeng ${ }^{1}$, Zeng Meilin ${ }^{2}$, Li Ke $^{1}$, Fang Xiaojuan $^{3}$, Xu Wenlei $^{3}$ \\ ${ }^{1}$ Nanchang institute of technology, Nanchan, 330013, China \\ ${ }^{2}$ Jiangxi Vocational Technical College of Industry Trade, Nanchang, 330000, China \\ ${ }^{3}$ Nanchang DanXun electronic technology co. Ltd, Nanchang, 330000, China \\ axqqxqqxqq4228@126.com
}

Keywords: WIFI, LED Lighting, Wireless Communication, STC12C5202AD

\begin{abstract}
Along with the national energy conservation policy for the deepening of LED lighting to replace the traditional incandescent lighting has become mainstream, but at the same time the development of wireless communication technology makes the LED lighting unit individual nodes of the network into reality. To this end, proposes a LED lighting system based on the network environment, the system uses STC12C5202AD microcontroller as the core, through the WIFI wireless communication module ESP8266 as the data transmission, so as to achieve the ULN2987 chip for the LED lighting system and its control drive. The experimental results show that the average packet loss rate is less than $5 \%$, the average time is less than $2 \mathrm{~ms}$ in $10 \mathrm{~m}$, and to meet the functional design requirements.
\end{abstract}

\section{Introduction}

Due to the short service life of conventional lamps, the consumption of electricity is very large, and the types of control methods are very small; on the other hand, the illumination of lower power lighting lamps is too low to meet the lighting requirements, while the higher power energy-saving lamp lighting The high energy consumption of the light is not conducive to energy-saving control; the attenuation of energy-saving light is very serious, and the longer the usage time is, the lower the lighting of the energy-saving lamp is, and the light emitted by the energy-saving lamp is less and less as the use time increases. Therefore, traditional lighting has gradually been replaced by LED lamps that have appeared in the later period, and LED lamps have many advantages such as energy saving, low light attenuation, and flexible types of control methods, which are widely used in various lighting systems in society. Because the application of Wi-Fi technology in home control systems has become more and more mature, people have begun to apply Wi-Fi technology to industrial control systems, wireless lighting control systems and smart positioning systems for large-scale applications, and achieved good results, such as wireless lighting. With the application of control systems, people can control hundreds of lights on and off at regular intervals through the Wi-Fi network, improving control efficiency. At the same time, but also reduce energy consumption plays an important role.

This design incorporates the flexibility of the Wi-Fi network [1-3] and the superior lighting 
characteristics of the LED lighting. An indoor wireless lighting control system is designed: the system consists of a control terminal, routers, terminal nodes, and LED lights. Constituted with the WIFI system, first of all we will make up the network of routers, terminal nodes, LED lights, Wi-Fi systems, and then the control terminal sends command information to the above network, and finally realizes LED control of each node. From the entire design perspective, the system has functions such as remote adjustment of light intensity and remote switch control, which solves the problems of fewer types of traditional lighting control methods, poor operational procedures, and high power consumption, and therefore can be seen from actual application results. Out, this design has a high value of application and promotion value, while changing the traditional lighting concept, it will also create great economic benefits.

\section{Overall design}

The entire system is composed of three major parts: the control side, routers and terminal nodes. The control side consists of a single-chip microcomputer and a Wi-Fi module. The main capability is to send control command information and receive the data information returned by the terminal node; the router is used to establish Wi-Fi. The main function of the network is to make the command information sent by the control terminal continue to transmit and forward the data information returned by the terminal node at the same time; the terminal node is composed of a single chip computer, a Wi-Fi module and an LED lamp, and is responsible for receiving the command information of the control terminal and converting them into corresponding The results of the control command, real-time control of the access of the LED lights, and the current status is returned to the remote control. The overall structure of the system shown in Figure 1.

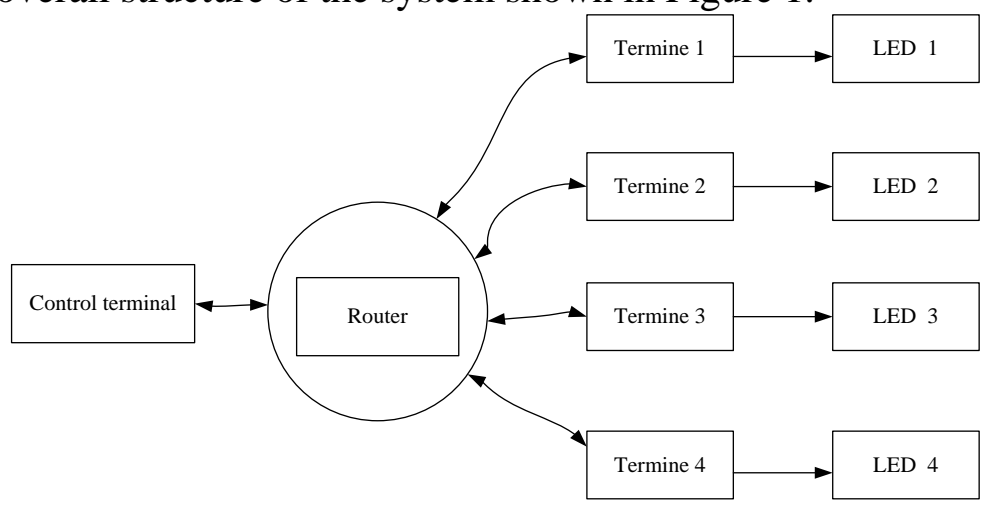

Figure 1 System Block Diagram

\section{3. hardware design}

\subsection{Hardware Composition and Working Principle}

According to the overall structure of the system design, the hardware of the system is composed of two parts: the control terminal and the terminal node. The control terminal consists of the minimum system composed of the MCU ( Microcontroller Unit ) STC12C5202AD, the LED lamp information generating circuit, the key circuit and the Wi-Fi communication circuit. The main function of the control terminal is to send The status information of the light control command, and receives the information returned by the terminal node, and judges the sending of the command information, and the terminal node is composed of a minimum system composed of the MCU STC12C5202AD, an LED switch circuit, and a Wi-Fi communication circuit. The function is to send the command information through the router control terminal, and control the working status of the 
LED lamp according to the control command information.

After the control terminal and the terminal node are powered on, they first set the initial state of their respective Wi-Fi modules, in which the control terminal is set to the TCP Server mode, the client is set to the Client mode, the initial setting is completed, and the control terminal controls the input circuit by the key switch The initial working state of the terminal node LED lamp is set. After the setting is completed, the control command information is sent through the module ESP2688. After the corresponding node receives the control command information, the command information is analyzed; according to the result of the analysis, the result can control the LED lamp. After the control is completed, the result of the execution of the command information is sent to the control terminal through the ESP8266 module, which is used to complete the reply to the control command information. In summary, the cycle of one data information communication is completed, and other cycles are also It will be executed in accordance with the above-mentioned working process of the cycle, and the process will continue until the transmission of data between the control end or the end node is interrupted.

\subsection{Key Circuit Design}

\subsubsection{STC89C52 minimum system circuit design}

The MCU STC12C5202AD is because of the high-speed one-chip computer that Taiwan Hong Jing Company designs to have the speed of $1 \mathrm{~T}$ to run, its running speed is 10 times that of ordinary C51 one-chip computer. The MCU STC12C5202AD uses the SOIC technology to capsulate, it has 20 pins, among them chip has 16 general-purpose input/output pins, an asynchronous communication serial port, 2 external interrupt input pins, 3 common timings/ Counter composition. From the pin number and have the function of view, the performance of the MCU STC12C5202AD is very superior, basically to meet the needs of various industrial control applications. The minimum system circuit formed by the MCU STC12C5202AD shown in Figure 2.

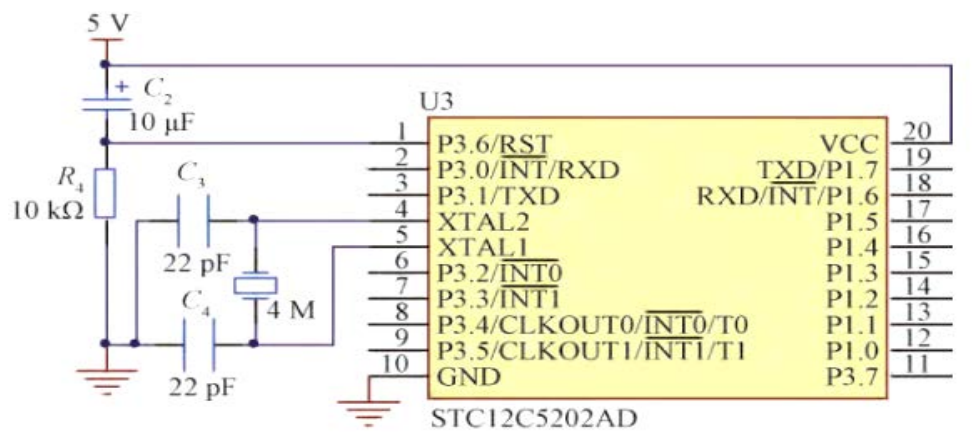

Figure 2 MCU STC12C5202AD minimum system circuit

The above minimum system circuit is mainly composed of a crystal control circuit and a reset circuit, the former adopts a crystal oscillation frequency of $4 \mathrm{MHz}$, and the starting capacitors C4 and C3 select $22 \mathrm{pF}$. The crystal oscillator control circuit generates a clock of a machine cycle which is a reference clock of the circuit. The clock can reference the information control, and the reset circuit adopts the control method of power-on reset, and the capacitor C2 and the resistor R4 together constitute a power-on reset circuit, and the software can only work normally after the hardware reset is completed.

Figure 2 shows that from the control point of view, the P3.0 pin and the P3.1 pins of the MCU are connected to the serial port pins of the ESP8266 to implement the information communication between the microcontroller and the module. The P3.2 pin of the MCU is connected with the reset 
pin of the module, and is used for realizing the reset control of the module. The P1.3 P1.7 pins of the MCU are connected with 5 independent key switches to realize the control of the working status of the LED lights of the terminal nodes. P1.0 P1.2 and P3.7 pins are connected with 4 LED lights to indicate the running status of the host. From the terminal's point of view, the P3.0 pin and P3.1 pins of the MCU Connected to the serial port pins of the ESP8266 to implement information communication between the MCU and the module. The P3.2 pin of the MCU is connected with the reset pin of the module, and is used for realizing the reset control of the module. The P1.0 P1.7 pins of the MCU are connected to the input of the ULN2987, and are used to control the eight lamp beads of one LED lamp. The P3.7 pin of the MCU is connected to a LED indicator for prompting to receive the data information sent by the host.

Wi-Fi Communication Circuit Design

ESP8266 is a Wi-Fi module with ultra-low power consumption [4-7], the module has a serial port transparent transmission function, can achieve transparent transmission through the control issued AT command, which greatly reduces the number of variables in the software. Designed for the practical application of the mobile Internet of Things, the user's controlled device can be involved in the Wi-Fi network, and the local area network or the Internet can control the access device. The communication between the STC12C5202AD and the ESP8266 is performed through a serial port. The pin is connected to the TXD pin of the module, and the pin P3.1 is connected to the RXD pin. The P3.2 pin of the MCU is connected to the reset pin of the module to implement the reset control of the module. The reset signal is active to the low level.

ESP8266 communication circuit is shown as in Figure 3.

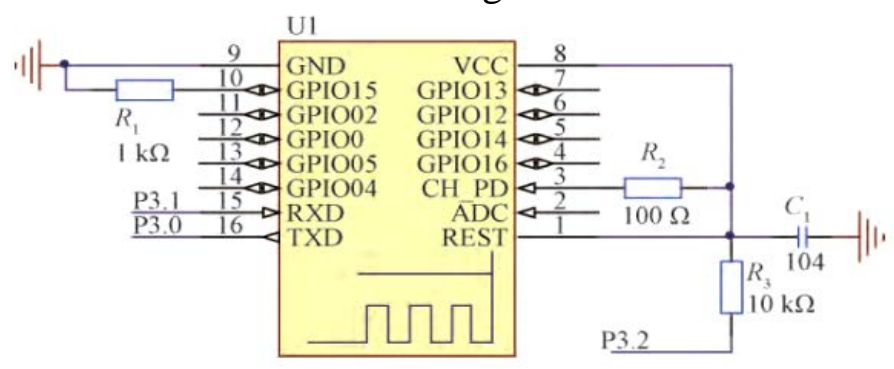

Figure 3 ESP8266 communication circuit

\subsubsection{Terminal node LED lamp control circuit design}

The LED lamp control circuit can realize the control of 8 LED lamp beads in a LED lamp, and the circuit can use the PWM dimming method to adjust the brightness of the 8 LED lamps under the control of the software. Added an LED indicator for operation indication information to display the status of data transmission and reception.

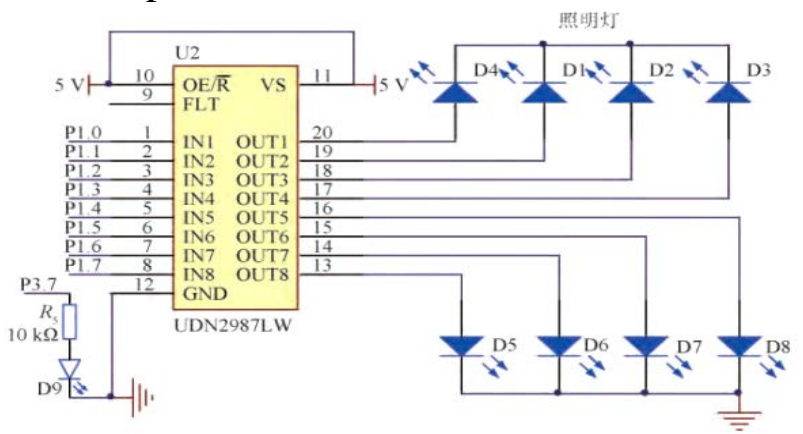

Figure 4 LED lighting control circuit 
The indicating circuit for receiving and sending data information is connected to the P3.7 pin of the MCU. When sending data information and receiving data, the MCU controls the P3.7 pin to output low level to realize the indication of data information sending and receiving; the LED light controls The circuit [8-10] adopts UDN2987 to control, its 8 inputs are connected to P1 mouth of the MCU respectively, when P1 mouth exports the high level, 8 LED lamp beads are lighted up, when P1 mouth exports the low level, 8 LED lights are extinguished.

LED light control circuit shown in Figure 4.

\section{Software Design}

In the above system, the software is designed to be used in the Wi-Fi controlled LED lighting system. The software consists of two parts: (1) Remote control function, and the command status of the terminal's LED lighting is performed through the control terminal sending command information. Control; (2) The local control function, the local need to achieve the function includes receiving the control terminal command information, and according to the control terminal under the command information control to change the working status of the LED lighting.

\subsection{Control side main flow design}

After the main program starts running, it needs to be initialized. The initialization process includes: initialization of the MCU I/O port and Wi-Fi module initialization; after the initialization is completed, the program loops through the condition loop body while(l) to execute the status display program, key program, Wi-Fi communication program, and detects the reset command information sent from the slave, resets the module if it receives the reset command information, and returns to the start of the loop body if it does not receive the reset command Figure 5 shows.

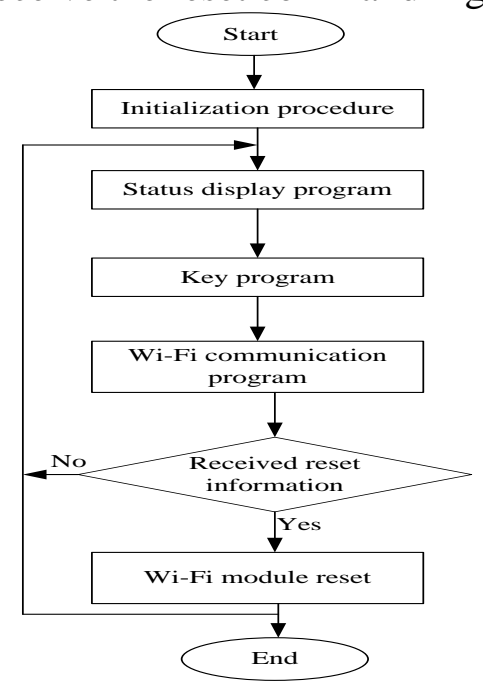

Figure 5 Software main flow chart

In the above flow chart figure, the communication program is divided into two parts, namely, the control terminal sending protocol and the terminal node reply command protocol. The control command information issued by the former control terminal transmits data information in hexadecimal format, and the frame starts with 0xFE. The receiving node IP address is 4 bits, the status word is 1 bit, the data is composed of 1 byte for representing the controlled brightness level, the end of the frame is represented by 0xAA, the last byte is the CRC8 checksum, and the latter is the sender's data information. Data information is transmitted in hexadecimal format. The frame starts with 0xFE, the receiving node IP address is 4 bits, the status word is 1 bit, and the data is 
composed of 1Byte for representing the brightness level of the control. The end of the frame is represented by 0xAA. The byte is a CRC8 checksum.

\subsection{Terminal node main program design}

The control method of the main program of the terminal node is similar to the control method of the main program of the control terminal, and the initialization operation is performed after the main program operation starts. The initialization process includes the initialization of the microcontroller I/O port and the initialization of the ESP8266 module, and after the initialization is completed, the program is in the conditional loop body. While(l) loops through the status display program, Wi-Fi communication program, and light control program; at the same time, it detects the reset command information sent from the slave, and if reset command information is received, resets the system module; if no reset command information is received Return to the beginning of the loop body to continue execution, where the light control program of the terminal node is executed in the terminal of $25 \mathrm{~ms}$ of timer 0 , when the interrupt is generated, the current brightness adjustment value is firstly judged, and if it is 1 value, it is $25 \mathrm{~ms}$. When the terminal is executed, the light is turned off, and the P1 port outputs the all-zero control command, and the other three modes of processing are the same as the brightness 1 , except that the value of the all-one value of the P1 port output becomes longer, and the value of the all-one value of the $\mathrm{P} 1$ port output is longer and higher. The longer the duration of the level is, the higher the brightness of the light is. With this type of PWM dimming, the brightness of the light is adjusted.

\section{Experimental results}

The specific tests in this article were conducted in an independent laboratory with a total length of 15 meters, a width of 7 meters, and a total area of 105 square meters. The obstacles will affect the Wi-Fi signal. In order to test the results more realistic, so this laboratory has a small amount of obstacles, the test is divided into 1 sets of 5 meters, 8 meters, 10 meters spacing 4 sets of different conditions for information transceiver control, the total number of times are 500 times, the test results show that within 10 meters, the average lost packet probability is below $2 \%$, the average time is less than 5 milliseconds, the best effect is within the radius of 8 meters, and there is a big delay beyond 10 meters. The probability of losing data packets is still low, and overall meets the functional design requirements. Detailed test results are shown in Table 1.

Table 1 Test result table

\begin{tabular}{cccc}
\hline $\begin{array}{c}\text { Test distance } \\
\text { Distance } / \mathrm{m}\end{array}$ & $\begin{array}{c}\text { Terminal successful } \\
\text { Responses/times }\end{array}$ & Total success rate/\% & Average time /m s \\
\hline 1 & 498 & 99.6 & 1.6 \\
5 & 496 & 99.2 & 2.8 \\
8 & 495 & 99.0 & 3.4 \\
10 & 491 & 98.2 & 4.9 \\
\hline
\end{tabular}

\section{Conclusion}

This article describes the design and implementation of LED lighting system based on Wi-Fi environment, and through the debugging of software and hardware, it can meet the requirements of design well. The experimental verification shows that the system has high precision recognition rate and good real-time working characteristics. The working status is stable and has a good use prospect. 


\section{References}

[1] Li Zongqing, Liu Zhongfu Wu Xuefu. Measurement and Control System for Wireless Smart Home Comfort Level[J]. Foreign Electronic Measurement Technology, 2016,35(11):103-107.

[2] Li Zhendong. Design of Intelligent LED Insecticidal Lamp Control System[J]. mechanical and electrical engineering technology.,2015(05):96-99.

[3] Wang Xijuan. Design of LED Intelligence Lighting Control System[J].Manufacturing automation, 2012, 33(12): 128-131.

[4] Lou Tingting, Pan Feng. Design of Smart LED Control System Based on Wireless Sensor Network[J]. Computer Measurement \& Control, 2015, 23(6):1990-1992.

[5] Hui Yanbo, Li Yongchao, Wang Li, Niu Qunfeng. The design of ultrasonic proximity alarm device[J], Electronic Measurement Technology, 2016, 39(05):120-122.

[6]Shan Binghua, Zhang Fengdeng. Temperature and Humidity detection system based on CAN Bus[J]. Electronic Measurement Technology, 2016, 39(05):123-126. 